Cite this: RSC Adv., 2014, 4, 26824

\title{
Formation of superconducting yttrium barium copper oxide using sulphur-containing templates $\uparrow$
}

\author{
Received 20th May 2014 \\ Accepted 6th June 2014 \\ DOI: $10.1039 / c 4 r a 04745 a$
}

www.rsc.org/advances

The formation of yttrium barium copper oxide (YBCO) via biotemplated routes is often plagued by unwanted stable intermediates, some of which arise from the template itself. Here we describe a method which allows sulphur-containing templates, such as proteins, to form superconducting YBCO which would have hitherto resulted in non-superconducting sulphated phases.

The rise of biotemplated syntheses for the creation of complex metal oxides has opened up a new field in oxide development and discovery. Biotemplates are natural polymers found in many forms including long chain polysaccharides, proteins and more complex structures such as DNA or viruses. The templating behaviour generally arises from the presence of suitable chelation sites which can uptake metal cations from solution, sequestering them for use in the creation of the final product. There are a large number of inorganic materials which can be made in this way and in general, providing suitable precursors and heating protocols can be found, the desired product can be synthesised. Biotemplated methods work extremely well for complex metal oxides, with many classes of ternary and quaternary functional oxides successfully synthesized, for example piezoelectrics, ${ }^{1,2}$ electrode materials ${ }^{3}$ and ferroelectrics. ${ }^{4}$ While solid state routes also work very well for these types of materials, they tend to involve lengthy processing times, which make them impractical and expensive. The main advantage of biopolymer-mediated synthesis is the significantly shorter synthesis times required to produce such materials, and the additional levels of morphological control offered.

${ }^{a}$ School of Chemistry, Cantock's Close, University of Bristol, Bristol, BS8 1TS, UK. E-mail: simon.hall@bristol.ac.uk

${ }^{b}$ Bristol Centre for Functional Nanomaterials, Centre for Nanoscience and Quantum Information, University of Bristol, Tyndall Avenue, Bristol, BS8 1FD, UK

${ }^{c}$ Nagaoka University of Technology, 16031 Kamitomioka, Nagaoka, Niigata 9402188 , Japan

$\dagger$ Electronic supplementary information (ESI) available: See DOI: $10.1039 / \mathrm{c} 4 \mathrm{ra} 04745 \mathrm{a}$
The high temperature superconductor (HTSC) yttrium barium copper oxide (YBCO) has been synthesized using biopolymers, with numerous novel morphologies developed purely as a result of the biopolymers used either through sacrificial templating methods, ${ }^{5}$ or through nanoscale crystal growth. ${ }^{6}$ There are a large number of templates which have been successfully employed to synthesise YBCO, including dextran, ${ }^{7}$ sodium alginate, ${ }^{8}$ and chitosan. ${ }^{9}$ The solid state techniques to create YBCO are plagued by the formation of recalcitrant phases, which are responsible for the long calcination times. In addition, the phase produced is generally poorly oxygenated, requiring additional annealing stages to create a good oxygen stoichiometry for superconducting properties. For $\mathrm{YBa}_{2} \mathrm{Cu}_{3} \mathrm{O}_{7-\delta}$ (Y123), a value of $\delta \approx 0.1$ gives the highest transition temperature. ${ }^{\mathbf{1 0}}$ Biotemplated methods generally give phase pure YBCO, and do not required an oxygen atmosphere or annealing step,,$^{5,7,11}$ and as such, represent a large improvement in both time and purity over the solid state methods.

Of all of these successful syntheses, however, there is one notable exception: the use of sulphur-containing templates such as $\kappa$-carrageenan. Previous work in this area concluded that the formation of YBCO was not possible due to the formation of highly stable barium sulphate (m.p. $1345{ }^{\circ} \mathrm{C}$ ) during the early part of the calcination process. ${ }^{12,13}$ Theoretically, around half of the barium reacted with the sulphur groups in the $\kappa$-carrageenan, leading to a significant reduction in the amount of barium carbonate which is responsible for initiating Y123 phase formation. This suppression of $\mathrm{BaCO}_{3}$ leads to the formation of other stable intermediates such as the nonsuperconducting "green phase" $\mathrm{Y}_{2} \mathrm{BaCuO}_{5}(\mathrm{Y} 211), \mathrm{Y}_{2} \mathrm{Cu}_{2} \mathrm{O}_{5}$, $\mathrm{CuO}$ and other barium deficient phases. As a result, it was concluded that sulphur-containing templates were not suitable for use with YBCO, effectively discounting many proteins (the amino acid cysteine contains a disulphide bridge) from being used for YBCO and other barium-containing complex oxides.

Megabalanus rosa cement protein (MRCP), produced by the Megabalanus rosa, or acorn barnacle, is one of a group of a relatively newly-isolated proteins, which are used by barnacles 
to adhere to rocks. Barnacles are well known for their ability to remain securely attached to rocks and other surfaces in the ocean, and being commonly found around the coastal regions, they must be able to withstand the force of breaking waves and tides in order to survive. The crystal structure of the protein is not yet known, however the composition has been found using reverse HPLC and cloning, ${ }^{14}$ and consists of a cysteine-rich, charged molecule, made up of 202 amino acids, the first 19 of which are thought to be used as a signal sequence, in the replication of the protein by the barnacle. The Cys residues are thought to be of great importance in maintaining exposed charged surfaces, which are reported to provide the interaction between barnacle and rock. These Cys residues could in theory provide chelation sites for introduced metal cations, although as above, the sulphur content would appear to preclude the use of MRCP and other cysteine-rich proteins in the synthesis of barium-containing materials. ${ }^{13}$ Here we report the first successful synthesis of Y123 using sulphated templates ( $\kappa$-carrageenan and MRCP) illustrating that sulphur-containing templates can be successfully employed for the synthesis of Y123 without the formation of $\mathrm{BaSO}_{4}$ impurity phases. The use of a protein template may also offer the opportunity to create chosen crystal morphologies designed from the bottom up, through judicious placement of particular chelating residues within the template molecule.

Stoichiometric quantities of yttrium, barium and copper nitrates (all Sigma Aldrich, UK) were dissolved in de-ionised water to form a solution with a $0.05: 0.1: 0.15 \mathrm{M}$ ratio. Separately, $\kappa$-carrageenan was dissolved in hot water in two different ratios: $0.15 \mathrm{~g}$ in $10 \mathrm{ml}$ of water (which will be referred to as "high ratio" samples), and $5 \mathrm{mg}$ in $10 \mathrm{ml}$ (the "low ratio" samples). The template was then vigorously mixed with the precursor solution in a ratio of $10 \mathrm{ml}: 2 \mathrm{ml}$ (template : precursor) to form gels. The samples prepared using MRCP used the low ratio, and the control sample was prepared as a $2 \mathrm{ml}$ sample of precursor containing no templating agent. The dried films were then calcined in air at $920^{\circ} \mathrm{C}$ for $2 \mathrm{~h}$ with a ramp rate of $10^{\circ} \mathrm{C} \mathrm{min}^{-1}$. The resulting black powders were then analysed using powder X-ray diffraction (PXRD) (D8 Advance, Bruker, Germany), scanning electron microscopy (SEM) (JSM 6330F, JEOL, Japan) and high resolution transmission electron microscopy (HRTEM), including selected area electron diffraction (JEM 2010, JEOL, Japan). Elemental content was determined using energy dispersive X-ray analysis (EDXA) (Oxford Cryosystems, UK) fitted to the HRTEM and SEM. Magnetometry was performed using a superconducting interference device (SQUID) (Magnetic Property Measurement System Quantum Design, USA). Samples measured were powdered and placed inside a gelatine capsule.

As-prepared samples were examined using PXRD in order to determine the crystallochemical composition of the materials, as shown in Fig. 1. In addition, selected area electron diffraction and EDXA were also performed in order to identify each phase within the sample directly. As has been shown previously, ${ }^{13}$ the sample containing a high ratio of $\kappa$-carrageenan-to-precursor (Fig. 1a) produced almost no Y123 phase, with $\mathrm{BaSO}_{4}$ (JCPDS card 01-076-0213) being the most prominent phase (ESI, Fig. S1a-c $\dagger$ ). $\mathrm{BaSO}_{4}$ forms between $300{ }^{\circ} \mathrm{C}$ and $400{ }^{\circ} \mathrm{C}$, (see ESI,
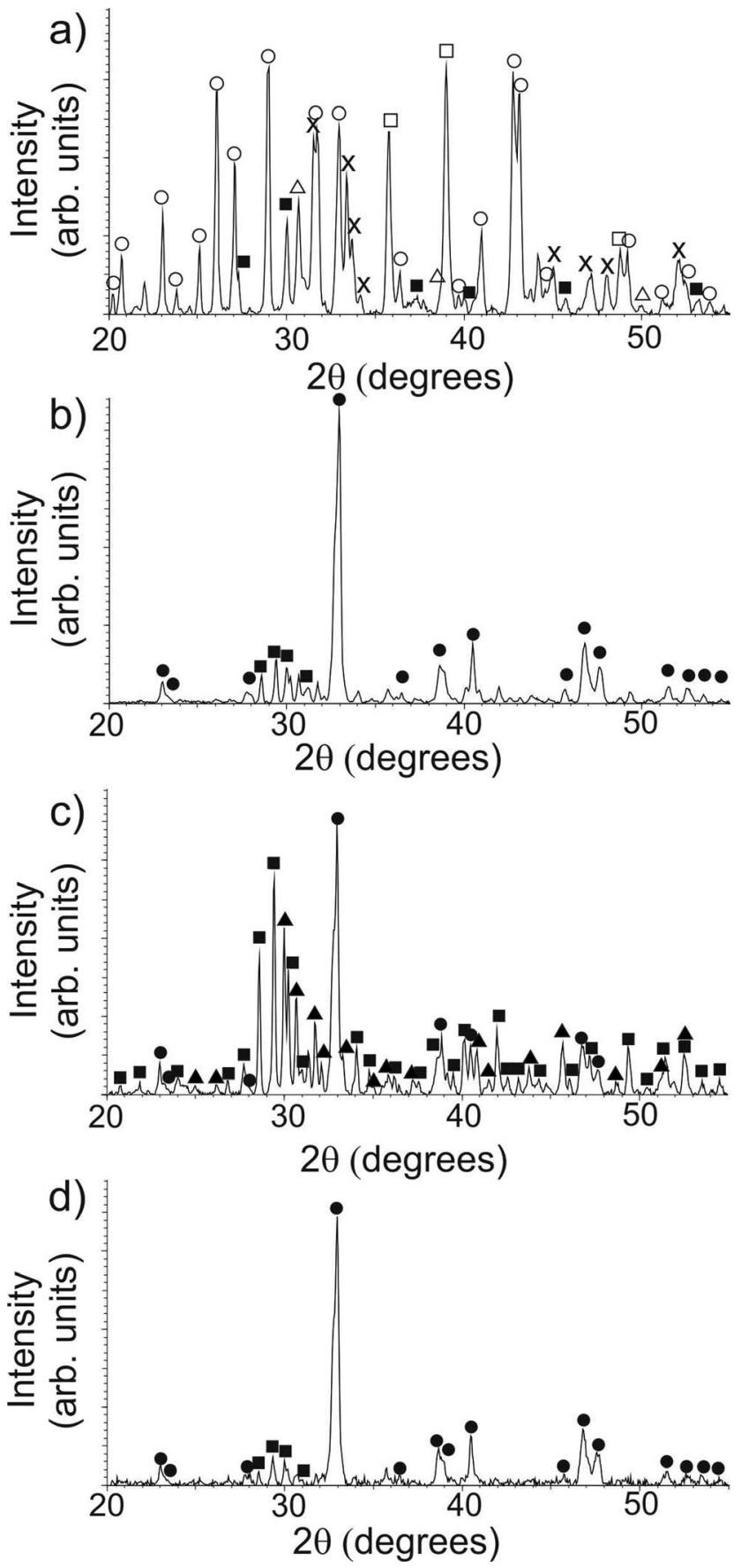

Fig. 1 PXRD of the different samples: (a) high-ratio $\kappa$-carrageenan; (b) low-ratio $\kappa$-carrageenan; (c) control sample containing no template; and (d) MRCP-templated sample. Phases are marked as $\mathrm{Y} 123(\mathbf{O})$, $\mathrm{BaCuO}_{2}(\boldsymbol{\square}), \mathrm{BaSO}_{4}(\mathrm{O}), \mathrm{Y}_{2} \mathrm{O}_{2} \mathrm{~S}(\Delta), \mathrm{CuO}(\square), \mathrm{Y} 211(\boldsymbol{\Delta})$ and $\mathrm{Y}_{2} \mathrm{Cu}_{2} \mathrm{O}_{5}(\times)$.

Fig. S2 $\dagger)$ and due to its high melting point $\left(1345{ }^{\circ} \mathrm{C}\right)$, remains solid in the reaction mixture at the final calcination temperature $\left(920{ }^{\circ} \mathrm{C}\right)$, thus making the majority of the barium ions unavailable for the formation of Y123. In addition to this, yttrium- and copper-rich phases such as $\mathrm{Y}_{2} \mathrm{Cu}_{2} \mathrm{O}_{5}$ (JCPDS card 01-083-0341, ESI, Fig. S1d-f $\dagger$ ), copper oxide (JCPDS card 01-0741021, ESI, Fig. S1g- $\mathrm{i} \dagger$ ) and $\mathrm{Y}_{2} \mathrm{O}_{2} \mathrm{~S}$ (JCPDS card 00-024-1424) were formed instead of the desired Y123 product, due to the 
unavailability of barium within the system. We then reduced the mass of $\kappa$-carrageenan used, until arriving at a biopolymerinorganic ratio where only a very low concentration of the barium ions are used in $\mathrm{BaSO}_{4}$ formation, and as such the desired Y123 product was able to form (JCPDS card 01-079-0318, ESI, Fig. S3†). This can be observed as a marked difference between the PXRD patterns for the low-ratio templated sample (Fig. 1b) and the high-ratio sample (Fig. 1a).

A control sample was also prepared using the same concentration of precursor ions but no template. Three major phases were observed: $\mathrm{BaCuO}_{2}$ (JCPDS card 01-070-0441, ESI, Fig. S4a-c $\dagger$ ), the non-superconducting Y211 (JCPDS card 01078-2214) (ESI, Fig. S4d-f $\dagger$ ), and Y123 (ESI, Fig. S4g-i†). By introducing a low ratio of templating material, the formation of the Y123 phase is favoured over these intermediates suggesting that the chelating ability of the template is providing enough spatial separation of ions to reduce the likelihood of the formation of large-scale intermediate crystallites, as was observed in the control sample.

The phases of the different materials present were also confirmed using HRTEM and selected area electron diffraction.
Fig. 2 shows HRTEM micrographs of the major phase in each sample with lattice planes indicated corresponding to each material: $\mathrm{BaSO}_{4}$ in the high ratio sample (Fig. 2a), Y123 in the low-ratio sample (Fig. 2b), $\mathrm{BaCuO}_{2}$ in the control sample (Fig. 2c) and Y123 in the sample templated using MRCP (Fig. 2d).

The samples were examined using SEM in order to investigate crystallite morphology. The samples prepared with high ratios of $\kappa$-carrageenan resulted in a reticulated, blocky morphology, as observed previously ${ }^{13}$ (Fig. 3a). Samples synthesised using a low ratio of $\kappa$-carrageenan also show this reticulated morphology (Fig. 3b), indicating that the templating effect is effective at directing the crystallite growth in the same way as a sample synthesized using a high ratio of template-toprecursor. The morphological similarities between the samples prepared using high and low ratios of template-to-precursor suggests that the template is having the same effect on crystal morphology in both cases regardless of the phase forming. Conversely the control sample is crystallographically and compositionally inhomogeneous (Fig. 3c). This is also evident in comparison between the particle sizes, which show that Y123
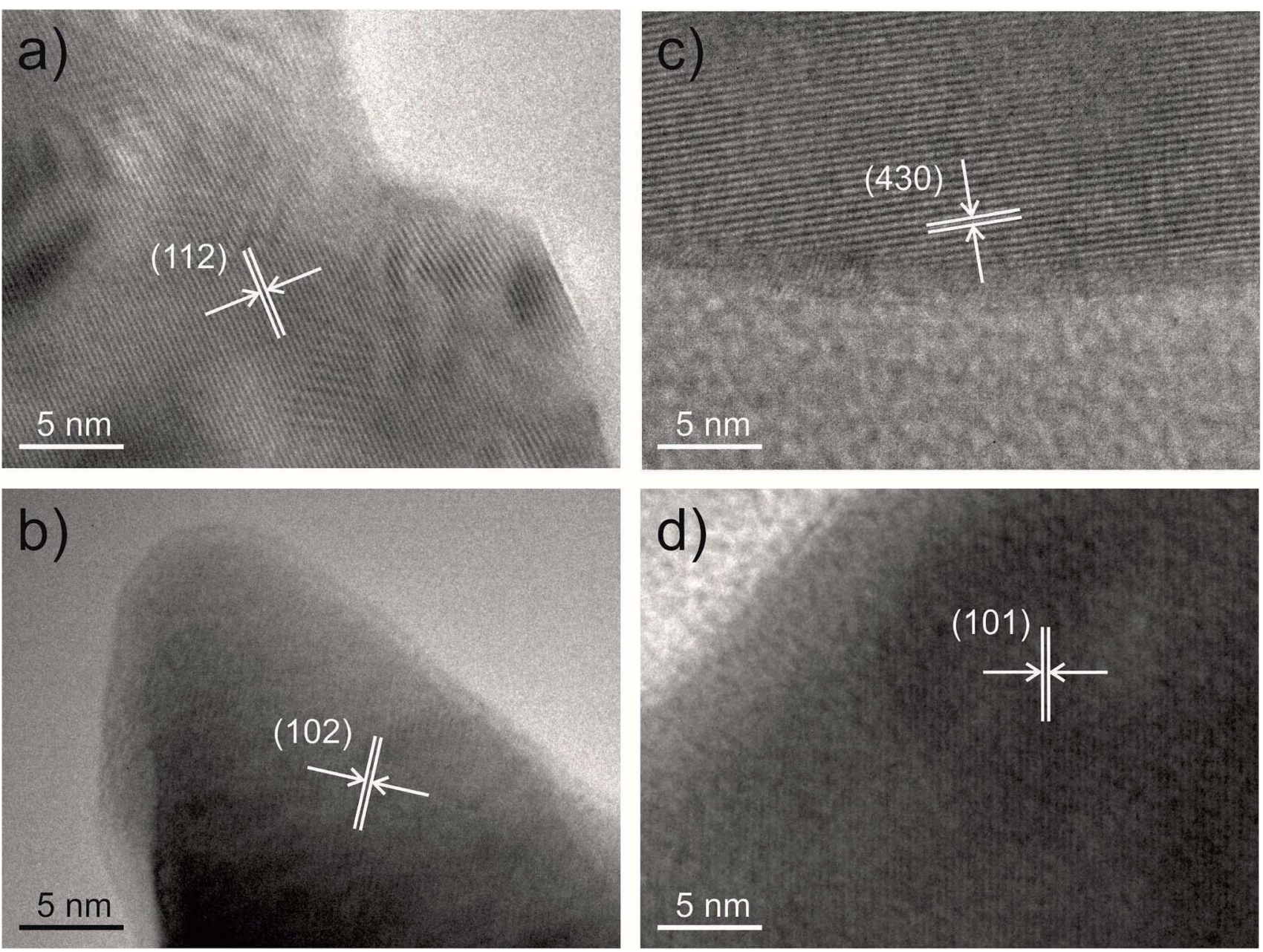

Fig. 2 TEM micrographs of the major phase in each sample. (a) the high-ratio sample with $\mathrm{BaSO}_{4}$ lattice planes highlighted; (b) the low-ratio sample with Y123 lattice planes highlighted; (c) the control sample with $\mathrm{BaCuO}_{2}$ lattice planes highlighted; and (d) the MRCP-templated sample with Y123 lattice planes highlighted. 


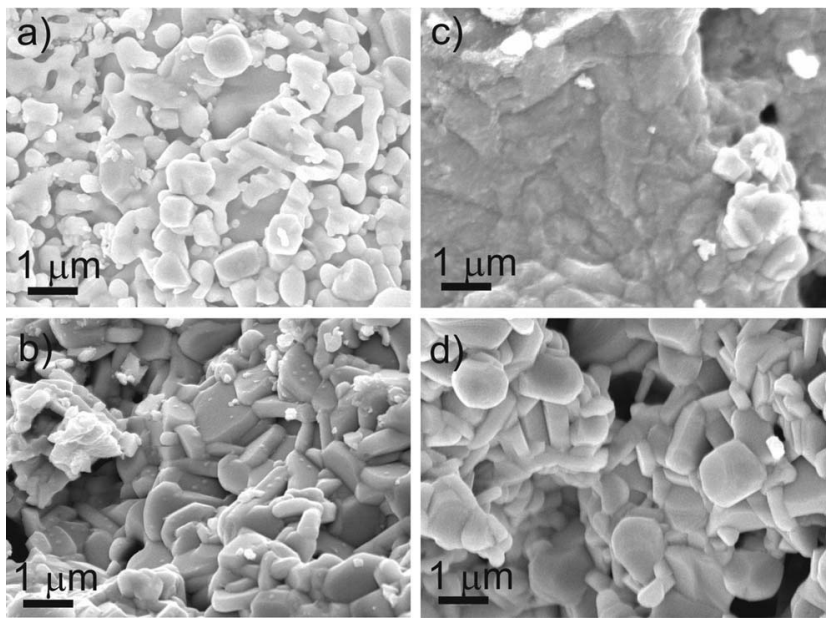

Fig. 3 SEM micrographs showing the morphologies found using different templates. (a) High ratio $\kappa$-carrageenan sample with similar reticulated blocky structure to (b) low-ratio of $\kappa$-carrageenan sample. (c) Crystallographically and morphologically inhomogeneous control sample. (d) The sample templated using MRCP with similar structure to the $\kappa$-carrageenan samples.

templated with both ratios of template produce crystallites with similar average sizes, as shown in Fig. 4. Samples templated using both high and low ratios gave particles with an average largest dimension of $0.91 \pm 0.9 \mu \mathrm{m}$ and $0.74 \pm 0.4 \mu \mathrm{m}$ respectively, as opposed to the control sample which showed a smaller average size of $0.21 \pm 0.1 \mu \mathrm{m}$. The inhomogeneous phase observed in the high-ratio $\kappa$-carrageenan The difference in particle size between the $\kappa$-carrageenan-templated samples and the control is indicative of the effect which the template is having on the morphology of the final product. The MRCPtemplated sample has a similar particle size to the $\kappa$-carrageenan samples, which indicates that the protein is having a templating effect on the morphology of the product. The observation that a sulphur-containing polysaccharide can provide a templating effect at low ratios allows us to use a cysteine-rich protein as a biotemplate for the first time. MRCP was used to create Y123 without the formation of significant or

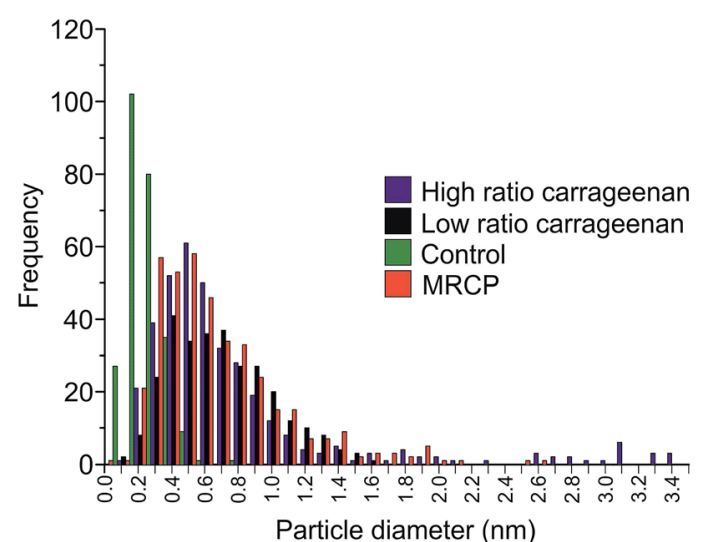

Fig. 4 Histogram plots of crystallite size, measured using the longest dimension of the crystallites. detrimental quantities of impurity phases. The PXRD pattern (Fig. 1d) indicates that the majority phase is Y123 (ESI, Fig. S5†), similar to the low-ratio $\kappa$-carrageenan samples. Fig. $2 \mathrm{~d}$ shows a HRTEM image illustrating Y123 lattice planes.

The morphological structure of the crystallites was found to be reticulated and blocky, indicating a similar structuredirecting effect on the Y123 phase (Fig. 3d), as was observed in the low ratio $\kappa$-carrageenan. The average particle size for MRCPtemplated YBCO was found to be $0.73 \pm 0.3 \mu \mathrm{m}$, similar to the $\kappa$ carrageenan samples, and indicates that the template is having an effect on the size of crystallites formed.

SQUID magnetometry was performed on all samples containing the Y123 phase in order to determine the superconducting transition temperature and volume susceptibility (Fig. 5). All of the transitions are broad due to the samples being powdered and highly polycrystalline. The transition temperatures of the samples varied slightly, with the control sample giving the highest $T_{\mathrm{c}}$ of $93 \mathrm{~K}$, followed by the low ratio $\kappa-$ carrageenan-templated sample with a $T_{\mathrm{c}}$ of $89.5 \mathrm{~K}$, and the MRCP-templated sample showing a transition at $89 \mathrm{~K}$. Although the $T_{\mathrm{c}}$ of the MRCP sample is the lowest, under zero-field cooling the volume susceptibility, $\chi_{\mathrm{v}}$, was found to be the most negative, indicating, to a first approximation, that $21.0 \%$ of the sample was in the superconducting state below the transition temperature. This is comparable to other Y123 samples synthesised using biotemplates, ${ }^{5,7}$ and also shows a significantly higher percentage than the low ratio $\kappa$-carrageenan-templated sample, of which approximately $12.5 \%$ is superconducting. The lowest volume fraction was in the control sample, showing around $7.5 \%$ of the material as superconducting phase, something which would be expected from the PXRD data which indicated a large number of impurity phases. This last result shows the general efficacy of biotemplates in the improved synthesis of YBCO.

In conclusion we have presented the first biotemplating of Y123 using sulphur-containing templates, including $\kappa$-carrageenan, which has previously been shown to be unsuitable for use in the synthesis of materials containing barium, due to the formation of barium sulphate. This synthesis method now allows for all the previously unusable sulphur-rich proteins to

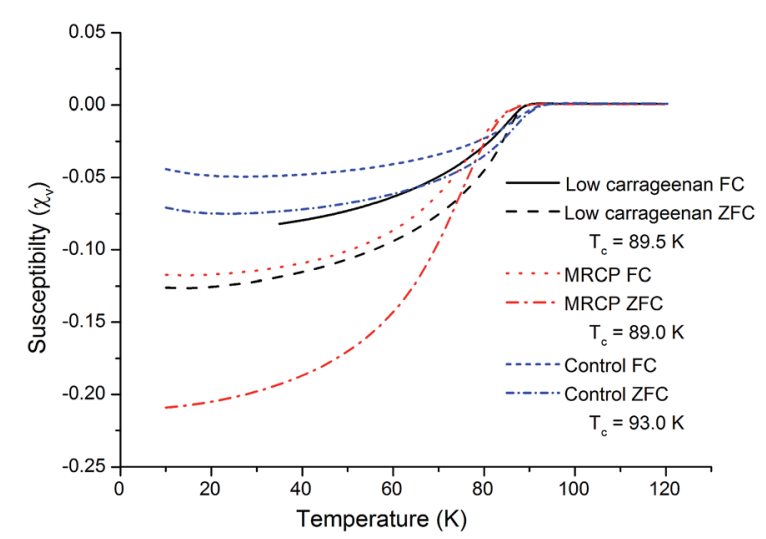

Fig. 5 SQUID magnetometry of the control sample, and samples prepared using low ratios of $\kappa$-carrageenan and MRCP. 
be considered as templates, and we have shown that they can be used to direct crystal size in the same way as has been observed in glucose-based templates. We have shown that this method is successful using the protein MRCP, which showed an increase in $\chi_{\mathrm{v}}$ over similar $\kappa$-carrageenan- prepared samples. Proteins, therefore, provide a new source of templates for complex materials containing barium, and similar metals, and through bottom-up design of chelation sites, may provide a means of creating previously inaccessible morphologies in complex materials.

\section{Acknowledgements}

We acknowledge the Electron and Scanning Probe Microscopy Facility at the University of Bristol for the use of SEMs and TEMs. RB and SRH acknowledge the Engineering and Physical Sciences Research Council (EPSRC), UK (grant EP/G036780/1), and the Bristol Centre for Functional Nanomaterials for project funding, and Kei Kamino and Yosuke Shida for providing and purifying the MRCP.

\section{References}

1 K. Cung, B. J. Han, T. D. Nguyen, S. Mao, Y.-W. Yeh, S. Xu, R. R. Naik, G. Poirier, N. Yao, P. K. Purohit and M. C. McAlpine, Nano Lett., 2013, 13, 6197.
2 Z. Schnepp, J. Mitchells, S. Mann and S. R. Hall, Chem. Commun., 2010, 46, 4887.

3 Y. Xia, W. Zhang, Z. Xiao, H. Huang, H. Zeng, X. Chen, F. Chen, Y. Gan and X. Tao, J. Mater. Chem., 2012, 22, 9209.

4 B. Li, J. Zhou, R. Zong, M. Fu, Y. Bai, L. Li and Q. Li, J. Am. Ceram. Soc., 2006, 89, 2298.

5 R. Boston, A. Carrington, D. Walsh and S. R. Hall, CrystEngComm, 2013, 15, 3763.

6 Z. Schnepp, S. C. Wimbush, S. Mann and S. R. Hall, Adv. Mater., 2008, 20, 1782.

7 D. Walsh, S. C. Wimbush and S. R. Hall, Chem. Mater., 2007, 19, 647.

8 Z. Schnepp, S. C. Wimbush, S. Mann and S. R. Hall, CrystEngComm, 2010, 12, 1410.

9 S. R. Hall, Adv. Mater., 2006, 18, 487.

10 R. Liang, D. A. Bonn and W. N. Hardy, Phys. Rev. B: Condens. Matter Mater. Phys., 2006, 73, 180505.

11 Z. Zhang, S. C. Wimbush, A. Kursumovic, H. Wang, J. H. Lee, H. Suo and J. L. MacManus-Driscoll, CrystEngComm, 2012, 14, 5765.

12 S. R. Hall, C. F. Hall, K. Hansberry, S. C. Wimbush, Y. Shida and W. Ogasawara, Supercond. Sci. Technol., 2012, 25, 035009.

13 E. Smith, Z. Schnepp, S. C. Wimbush and S. R. Hall, Phys $C$ Supercond., 2008, 468, 2283.

14 K. Kamino, Biochem. J., 2001, 356, 503. 